\title{
ON BOOLEAN ALGEBRAS OF PROJECTIONS AND SCALAR-TYPE SPECTRAL OPERATORS
}

\author{
W. RICKER
}

\begin{abstract}
It is shown that the weakly closed operator algebra generated by an equicontinuous o-complete Boolean algebra of projections on a quasi-complete locally convex space consists entirely of scalar-type operators. This extends $W$. Bade's well-known theorem that the same assertion is valid for Banach spaces; however, the technique of proof here differs from his method, which extends only to metrizable spaces.
\end{abstract}

In [1], W. Badé showed that every operator in the weakly closed operator algebra generated by a $\sigma$-complete Boolean algebra of projections on a Banach space is a scalar-type spectral operator (in the sense of Dunford, [2]).

The aim of this note is to show that this result can be extended to locally convex spaces. The methods used by Badé are not available, because they make use of the fact that the weak operator closure of the algebra generated by a $\sigma$-complete Boolean algebra of projections on a Banach space is again an algebra; the corresponding assertion is false for locally convex spaces in general. These methods are replaced by the theory of integration with respect to closed spectral measures (see §2).

Let $X$ be a locally convex Hausdorff space. The space $X$ will always be assumed to be quasi-complete. Let $L(X)$ denote the space of all continuous linear operators on $X$, equipped with the topology of pointwise convergence.

Theorem. Let the space $L(X)$ be quasi-complete. Let $\mathbb{Q}$ be an equicontinuous, o-complete Boolean algebra of projections in $L(X)$. Then every operator in the weak operator closure of the algebra generated by $Q$ is a scalar-type spectral operator.

1. Preliminaries. The dual space of $X$ is denoted by $X^{\prime}$. If $q$ is a continuous seminorm on $X$, let

$$
U_{q}^{\circ}=\left\{x^{\prime} \in X^{\prime} ;\left|\left\langle x, x^{\prime}\right\rangle\right| \leqslant 1 \text { for all } x \text { such that } q(x) \leqslant 1\right\} .
$$

An $X$-valued vector measure is a $\sigma$-additive map $m: \mathfrak{R} \rightarrow X$ whose domain $\mathscr{N}$ is a $\sigma$-algebra of subsets of a set $\Omega$. For each $x \in X^{\prime}$, the complex-valued measure $E \mapsto\left\langle m(E), x^{\prime}\right\rangle, E \in \mathscr{T}$, is denoted by $\left\langle m, x^{\prime}\right\rangle$.

If $q$ is a continuous seminorm on $X$, then the $q$-semivariation, $q(m)$, of $m$ is the set function defined by

$$
q(m)(E)=\sup \left\{\left|\left\langle m, x^{\prime}\right\rangle\right|(E) ; x^{\prime} \in U_{q}^{\circ}\right\}, \quad E \in \mathfrak{R} .
$$

Received by the editors January 6, 1982 and, in revised form, April 16, 1982.

1980 Mathematics Subject Classification. Primary 47B40; Secondary 46G10, 47D30.

Key words and phrases. Closed measure, spectral measure, scalar-type spectral operator, Boolean algebra of projections. 
For each $E \in \mathscr{R}$, the inequalities

$$
\begin{aligned}
\sup \{q(m(F)) & ; F \in \mathfrak{K}, F \subseteq E\} \\
& \leqslant q(m)(E) \leqslant 4 \sup \{q(m(F)) ; F \in \mathfrak{R}, F \subseteq E\}
\end{aligned}
$$

hold [3, Lemma II 1.2].

A complex-valued, $\Re$-measurable function $f$ on $\Omega$ is said to be $m$-integrable if it is integrable with respect to every measure $\left\langle m, x^{\prime}\right\rangle, x^{\prime} \in X^{\prime}$, and if, for every $E \in \mathfrak{N}$, there exists an element $\int_{E} f d m$ of $X$ such that

$$
\left\langle\int_{E} f d m, x^{\prime}\right\rangle=\int_{E} f d\left\langle m, x^{\prime}\right\rangle
$$

for each $x^{\prime} \in X^{\prime}$. If $f$ is an $m$-integrable function, then the mapping $f m: \mathfrak{N} \rightarrow X$ specified by

$$
(f m)(E)=\int_{E} f d m, \quad E \in \mathfrak{T},
$$

is called the indefinite integral of the function $f$ with respect to the measure $m$. The Orlicz-Pettis lemma implies that it is a vector measure. The element $(f m)(\Omega)$ of $X$ is denoted simply by $m(f)$.

The set of all $m$-integrable functions is denoted by $L(m)$. Members of $\Re$ are freely identified with their characteristic functions. An $m$-integrable function is said to be $m$-null if its indefinite integral is the zero vector measure. Two $m$-integrable functions $f$ and $g$ are $m$-equivalent or equal $m$-almost everywhere ( $m$ a.e.) if $|f-g|$ is $m$-null. The class of all $m$-integrable functions equivalent to a function $f \in L(m)$ is denoted by $[f]_{m}$.

Let $q$ be a continuous seminorm on $X$. If $f$ is an $m$-integrable function, then the $q$-upper integral, $q(m)(f)$, of $f$ is defined by

$$
q(m)(f)=q(f m)(\Omega) .
$$

For any continuous seminorm $q$ on $X$, the relations

$$
q(m)(f)=q(m)(|f|), \quad f \in L(m),
$$

and

$$
q(m)(f) \leqslant q(m)(g), \quad 0 \leqslant f \leqslant g ; f, g \in L(m),
$$

are valid [3, Lemma II 2.2]. Furthermore, the function

$$
f \mapsto q(m)(f), \quad f \in L(m),
$$

is a seminorm on $L(m)$.

Denote by $\tau(m)$ the topology on $L(m)$ which is defined by the family of seminorms (4), for every continuous seminorm $q$ on $X$. The resulting locally convex space is not necessary Hausdorff. The quotient space of $L(m)$ with respect to the subspace of all $m$-null functions is denoted by $L^{1}(m)$. The resulting Hausdorff topology on $L^{\prime}(m)$ is again denoted by $\tau(m)$. It is clear from $(1)$ that $\tau(m)$ is the topology of uniform convergence on $\mathfrak{N}$ of indefinite integrals. 
Since $X$ is quasi-complete, it follows that every bounded measurable function is $m$-integrable [3, Lemma II 3.1].

A set $E \in$ : $\Re$ is said to be $m$-null if $\chi_{E}$ is $m$-null. Two sets $E, F \in: \eta$ are $m$-equivalent if their characteristic functions are $m$-equivalent. Since $\left|\chi_{E}-\chi_{F}\right|=$ $\chi_{E \triangle F}$ where $E \triangle F=(E \cup F) \backslash E \cap F$, this is so if and only if $\chi_{t: \Delta F}$ is $m$-null. The set of all equivalence classes of $\mathscr{T}$ with respect to $m$-equivalence is denoted by $\because \pi(m)$.

The set ơ $(m)$ can be identified with the subset $\left\{\left[\chi_{t}\right]_{m} ; E \in\right.$ OR $\}$ of $L^{1}(m)$. Furthermore, as

$$
q(m)(E)=q(m)\left(\chi_{E}\right), \quad E \in \mathfrak{R},
$$

for each continuous seminorm $q$ on $X$, the topology and uniform structure $\tau(m)$ has a natural restriction to $\mathfrak{N}(m)$ which is again denoted by $\tau(m)$.

A vector measure $m$ is said to be closed if $\mathfrak{N}(m)$ is a complete space with respect to the uniform structure $\tau(m)$.

The following result, which is a slight extension of Theorem IV 4.1 of [3], shows that the closedness of a vector measure is a desirable property.

Proposition 1. Let $m: \mathcal{M} \rightarrow X$ be a vector measure. Then $m$ is closed if and only if $L^{\prime}(m)$ is complete.

Proof. If $L^{\prime}(m)$ is complete, then $m$ is closed [3, Theorem IV 4.1].

Suppose that $m$ is closed. If $\hat{X}$ denotes the completion of $X$ and $\hat{m}: \cdots \rightarrow \hat{X}$ the measure defined by $\hat{m}(E)=m(E), E \in \mathcal{N}$, then $\hat{m}$ is also closed. It is clear that $L^{\prime}(m) \subseteq L^{\prime}(\hat{m})$ and that $\tau(\hat{m})$ induces the $\tau(m)$ topology on $L^{\prime}(m)$. Since $L^{\prime}(\hat{m})$ is complete [3, Theorem IV 4.1], the proof is completed by showing that $L^{1}(m)=L^{1}(\hat{m})$.

It suffices to show that each $\hat{m}$-integrable function $f \geqslant 0$ is $m$-integrable. Choose simple functions $s_{n} \geqslant 0, n=1,2, \ldots$, such that $s_{n} \uparrow f$ pointwise. Since $\left(s_{n} \hat{m}\right)(E) \in X$ for each $E \in M, n=1,2, \ldots$, it follows from the Dominated Convergence Theorem and the quasi-completeness of $X$ that $(f \hat{m})(E) \in X$ for all $E \in \mathcal{u}$. Hence, $f$ is $m$-integrable.

2. Closed spectral measures. If $N$ is an equicontinuous subset of $X^{\prime}$, then $q_{N}$ denotes the continuous seminorm on $X$ defined by

$$
q_{N}(x)=\sup \left\{\left|\left\langle x, x^{\prime}\right\rangle\right| ; x^{\prime} \in N\right\}, \quad x \in X .
$$

For $x \in X$ and an equicontinuous subset $N$ of $X^{\prime}$, let $q_{N}^{x}$ denote the continuous seminorm on $L(X)$ given by

$$
q_{N}^{x}(T)=q_{N}(T(x)), \quad T \in L(\ddot{X}),
$$

where $q_{N}$ is the seminorm (5). The collection of all such seminorms $q_{N}^{x}$ determines the topology of $L(X)$.

A spectral measure in $X$ is an $L(X)$-valued, $\sigma$-additive and multiplicative map $P$ : $\mathfrak{N} \rightarrow L(X)$, whose domain $\mathfrak{N}$ is a $\sigma$-algebra of subsets of a set $\Omega$ such that $P(\Omega)$ is the identity operator. Of course, the multiplicativity of $P$ means that $P(E \cap F)=$ $P(E) P(F)$, for every $E \in \mathfrak{N}$ and $F \in \mathfrak{N}$. Since $L(X)$ is itself a locally convex space it is clear that spectral measures are vector measures. 
A spectral measure $P$ in $X$ is said to be equicontinuous if its range

$$
R(P)=\{P(E) ; E \in \mathfrak{N}\},
$$

is an equicontinuous part of $L(X)$. For such spectral measures, every bounded measurable function is $P$-integrable (see $\$ 1$ of [4]).

Let $P: \mathfrak{R} \rightarrow L(X)$ be an equicontinuous spectral measure. Let $f$ be a $P$-integrable function. It is a consequence of the multiplicativity of $P$ that the operator $P(f)$ commutes with $P(E)$, for every $E \in \mathfrak{R}$. Furthermore, the indefinite integral of the function $f$ with respect to $P$ is given by

$$
(f P)(E)=P(f) P(E), \quad E \in \mathfrak{N} \text {. }
$$

LEMMA 1. Let $P: \mathfrak{T} \rightarrow L(X)$ be an equicontinuous spectral measure. If $x \in X$ and $N$ is an equicontinuous subset of $X^{\prime}$, then there exists an equicontinuous subset $H$ of $X^{\prime}$, depending on $P$ and $N$, such that

$$
q_{N}^{x}(P(f)) \leqslant q_{N}^{x}(P)(f) \leqslant q_{H}^{x}(P(f)),
$$

for every $f \in L^{\prime}(P)$.

Proof. If $f$ is $P$-integrable, then a simple calculation using the definition of the $q_{N}^{x}$-upper integral of $f$ with respect to $P$, the formula (6) and the inequality (1) shows that

$$
q_{N}^{x}(P(f)) \leqslant q_{N}^{x}(P)(f) \leqslant 4 \sup \left\{q_{N}^{x}(T) ; T \in R(f P)\right\},
$$

where $R(f P)=\{P(f) P(E) ; E \in \mathscr{R}\}$ is the range of $f P$. Let $H$ denote the equicontinuous subset $\left\{4 P(E)^{\prime}\left(x^{\prime}\right) ; E \in \mathscr{T}, x^{\prime} \in N\right\}$ of $X^{\prime}$ (if $T \in L(X)$, then $T^{\prime}$ denotes the adjoint operator). It follows that

$$
4 q_{N}^{x}(T) \leqslant q_{H}^{x}(P(f)), \quad T \in R(f P) .
$$

The required inequality is clear from (7) and (8).

Let $P: \mathfrak{R} \rightarrow L(X)$ be an equicontinuous spectral measure. Then define

$$
g(P)=\left\{P(f) ; f \in L^{\prime}(P)\right\} .
$$

The identity (6) implies that the map $f \mapsto P(f), f \in L^{1}(P)$, is injective and hence, Lemma 1 implies that it is a linear homeomorphism of $L^{\prime}(P)$ onto $G(P)$. This fact together with Proposition 1 gives

Proposition 2. Let the space $L(X)$ be quasi-complete and let $P: \mathfrak{R} \rightarrow L(X)$ be an equicontinuous spectral measure. Then $P$ is a closed measure if and only if $Y(P)$ is complete for the relative topology induced by $L(X)$.

Proposition 3. An equicontinuous spectral measure $P: \mathfrak{R} \rightarrow L(X)$ is closed, if and only if, the range $R(P)$ of $P$ is a closed set in $L(X)$.

Proof. Suppose that $R(P)$ is closed. Let $\left(E_{\alpha}\right)_{\alpha \in A}$ be a Cauchy net in $(\mathscr{R}(P), \tau(P))$. If $q_{N}^{x}$ is a continuous seminorm for $L(X)$, then it follows from (2), the identity $\left|\chi_{E_{\alpha}}-\chi_{E_{\beta}}\right|=\chi_{E_{\mathrm{a}} \Delta E_{\beta}}$ and Lemma 1 that

$$
q_{N}\left(P\left(\chi_{E_{\alpha}}-\chi_{E_{\beta}}\right)(x)\right) \leqslant q_{N}^{x}(P)\left(E_{\alpha} \Delta E_{\beta}\right) \text {, }
$$


for each $\alpha, \beta \in A$. Also (3) and Lemma 1 imply that

$$
q_{N}\left(P\left(\chi_{E_{\alpha}}\right)(x)\right) \leqslant q_{N}^{x}(P)\left(\chi_{E_{\alpha}}\right) \leqslant q_{N}^{x}(P)(\Omega)<\infty,
$$

for each $\alpha \in A$. Since $X$ is quasi-complete and $R(P)$ is equicontinuous, it follows from (9) and (10) that there exists an operator $T \in L(X)$ such that $P\left(E_{\alpha}\right) \rightarrow T$, $\alpha \in A$. As $R(P)$ is closed, there is $E \in \Re$ such that $T=P(E)$. Then Lemma 1 implies that $E_{\alpha} \rightarrow E$ in $\Re(P)$. This shows that $\Re(P)$ is $\tau(P)$-complete.

Conversely, let $P$ be a closed measure. Let $\left(P\left(E_{\alpha}\right)\right)_{\alpha \in A}$ be a net in $R(P)$ which converges to $T \in L(X)$. If $q_{N}^{x}(P)$ is a continuous seminorm for $\tau(P)$, then there exists a continuous seminorm $q_{H}^{x}$ on $L(X)$ such that

$$
q_{N}^{x}(P)\left(E_{\alpha} \triangle E_{\beta}\right) \leqslant q_{H}^{x}\left(P\left(E_{\alpha}\right)-P\left(E_{\beta}\right)\right),
$$

for all $\alpha, \beta \in A$. As $\mathscr{R}(P)$ is complete, there exists $E \in \mathscr{N}$ such that $E_{\alpha} \rightarrow E$. Lemma 1 implies that $P\left(E_{\alpha}\right) \rightarrow P(E)$. It follows that $R(P)$ is closed.

An operator $T \in L(X)$ is called a scalar-type operator if there exists a spectral measure $P$ in $X$ such that $T \in \mathscr{G}(P)$.

3. Proof of the theorem. Let $K$ denote the closure of $Q$ in $L(X)$ and $\langle K\rangle$ denote the algebra generated by $K$ in $L(X)$. Interpreting $Q$ as the range of a spectral measure in $X$ on the Stone space of $Q$, it follows from Proposition 3.17 of [4] that $K$ is an equicontinuous, complete Boolean algebra of idempotents in $L(X)$. Accordingly, there exists an equicontinuous spectral measure $P$ in $X$ whose range is precisely $K$. Propositions 2 and 3 imply that $G(P)$ is closed in $L(X)$.

The algebra $\langle Q\rangle$ is a convex set. Hence, the weak operator closure of $\langle Q\rangle$ is the same as its (strong) closure in $L(X)$. Since the $L(X)$-closures of $\langle\mathscr{Q}\rangle$ and $\langle K\rangle$ are clearly equal, it suffices to show that any operator in the closure of $\langle K\rangle$ is a scalar-type operator.

Since $\langle K\rangle$ consists of all operators of the form $P(s)$, where $s$ is a $P$-simple function, it follows that $\langle K\rangle \subseteq g(P)$. The space $\mathscr{Q}(P)$ is always contained in the closure of $\langle K\rangle$ because the $P$-simple functions are $\tau(P)$-dense in $L^{1}(P)$. Since $\mathscr{G}(P)$ is closed (even complete) it follows that the closure of $\langle K\rangle$ is equal to $G(P)$.

It is worth noting that the algebra, $\langle Q\rangle$, generated by $Q$ is equal to the linear span of $Q$. Hence, although the weak (or strong) closure of $\langle Q\rangle$ may not be an algebra in $L(X)$, it is a closed subspace of $L(X)$.

ACKnowledgement. The author thanks Professor I. Kluvánek for valuable discussions.

\section{REFERENCES}

1. W. G. Badé, On Boolean algebras of projections and algebras of operators, Trans. Amer. Math. Soc. 80 (1955), 345-359.

2. N. Dunford and J. T. Schwartz, Linear operators, vol. III, Interscience, New York, 1971.

3. I. Kluvánek and G. Knowles, Vector measures and control systems, North-Holland, Amsterdam, 1976.

4. B. Walsh, Structure of spectral measures on locally convex spaces, Trans. Amer. Math. Soc. 120 (1965), $295-326$.

School of Mathematical Sciences, The Flinder's University of South Australia, Bedford PARK, 5042, Australia 